\title{
CBT for Culture Change: Formulating Teams to Improve Patient Care
}

\author{
Katherine Newman Taylor and Suzanne Sambrook \\ Southern Health NHS Foundation Trust, Southampton, UK
}

\begin{abstract}
Background: Increasingly, clinical psychologists and CBT trained clinicians work with and within teams. The cognitive model enables us to formulate the processes maintaining distress, and work with people to effect change. The model tends to be used to understand individuals' difficulties, but may be effective in making sense of problems within teams. Aims: This study aimed to (i) explore the value of the cognitive model in formulating key staff-service user relationships; and (ii) determine whether such an approach would yield useful team based interventions. Method: The cognitive interpersonal model was used to develop an idiosyncratic conceptualization of key staff-service user interactions in an inpatient setting. This then informed management team planning aimed at improving provision for service users, and staff experience. Additionally, frequency of challenging behaviours and levels of staff burnout were assessed before and after service changes, as preliminary outcome data. Results: The team formulation was effective in (i) making sense of interactions contributing to the maintenance of service users' challenging behaviours and staff burnout, and (ii) deriving systemic interventions likely to effect change. This was then used to guide service development planning. In support of a CBT approach to understanding and intervening with teams, preliminary data indicate that staff burnout and incidents of challenging behaviours reduced over time. Conclusion: The cognitive interpersonal model can be used to formulate relationships within teams and guide systemic change. This is likely to have a beneficial impact for both service users and staff.
\end{abstract}

Keywords: Formulating teams, challenging behaviour, burnout, cognitive interpersonal model.

\section{Introduction}

As clinical psychologists and CBT trained clinicians, many of us work with staff teams to improve services. The New Ways of Working documents (Lavender and Hope, 2007; Onyett, 2007) argue that one of the key roles of applied psychologists is to work with the system itself to improve team working and increase psychological understanding via training, supervision and support. The process of formulation in particular may help us to understand the interpersonal environment within teams, and derive effective interventions to improve the experience of service users and staff, and the effectiveness of these systems.

Many clinicians, particularly those in in-patient settings, work in stressful environments with an ongoing threat of aggression and other high risk behaviours. This can lead to staff

Reprint requests to Katherine Newman Taylor, Southern Health NHS Foundation Trust - Clinical Psychology, Grove House, Meridians Cross, Ocean Way, Southampton SO14 3TJ, UK. E-mail: knt@ soton.ac.uk 
burnout (Maslach and Leiter, 1997), with people feeling overwhelmed and exhausted, and resulting in disengagement with service users, which in turn affects quality of care (Currid, 2009). It has been suggested that it is more useful to consider team burnout than to focus on individuals, and to develop systemic interventions across staff groups if we are to address this problem effectively (Garman, Corrigan and Morris, 2002). Onyett (2007) suggests that peer consultation may be of particular value to staff working in in-patient or residential settings.

Although staff burnout can be detrimental to both staff and service users (Currid, 2009), it is not inevitable for clinicians working with high risk groups (Nelson, Johnson and Bebbington, 2009; Crawford, Adedeyi, Price and Rutter, 2010). It is essential therefore that those trained in conceptualizing the psychological processes that maintain distress, identify and address those factors that put individuals and staff teams at risk of burnout. A recent paper by Onyett (2011) suggests that sources of burnout are multidimensional and likely to include work overload, feeling undervalued, poor time management, poor role clarity, and lack of leadership. Problematic attitudes towards and expectations of service users, the team and management are also likely to contribute (O'Connell and Stein, 2011; Tsai, Salyers and McGuire, 2011).

One way of addressing this might be to formulate key processes in the system, to give a shared language and way of communicating complex issues, and to guide team based interventions. Onyett (2007) argues that "taking formulation into the wider context can be a powerful way of shifting culture(s)" (p 23). An awareness of protective factors is also likely to shape effective team interventions (Crawford et al., 2010).

Other approaches to facilitating change such as Appreciative Inquiry (AI; Cooperrider and Whitney, 2005) have been used in health service settings (Carr, 2009). AI focuses on building strengths within a team and collaborative problem solving. Interestingly, AI is based on a particular method of questioning similar to the Socratic process that underpins the development of cognitive formulation and intervention (Kowalski, 2008). AI's focus on the future can be very helpful, but (to date) fails to involve service user perspectives, and this is problematic if working towards a Recovery based approach to health care.

In individual CBT, formulation is central to understanding the development and maintenance of problems, and provides a basis for collaborative change work. Safran and Segal (1996) extend this primarily intrapersonal conceptualization to map out relationships between people. Their cognitive interpersonal model proposes that an individual's beliefs about other people will not only influence their own behaviour, but exert an effect on others (a "pull"), which is likely to lead the other person to act in such a way that the schema is confirmed. For example, if Alex believes that other people are rejecting her, interpersonal situations may be associated with anxiety and fear. Alex is likely to engage in a range of behaviours in order to manage her distress, such as failing to maintain eye contact or conversation with others, avoiding intimacy and declining social contact altogether. This will have an effect on Beth who is likely to reciprocate Alex's verbal and non-verbal cues, cease to make efforts to maintain closeness, and move away, thereby confirming Alex's original belief.

The management team for the in-patient unit involved in this study had expressed their wish to adopt a more patient-centred ethos, but were struggling to know how to effect such change. This provided a unique opportunity to explore the value of a cognitive approach to formulating team problems. This brief report aims to investigate the value of the cognitive interpersonal model in formulating staff-service user relationships, conceptualizing the team as a single entity (Garman et al., 2002), in order to derive interventions likely to improve 
the culture on the unit. We hypothesized that the team formulation would be effective in:

(i) making sense of interactions contributing to the maintenance of service users' challenging behaviours and staff burnout;

(ii) deriving systemic interventions likely to effect change.

Additionally, preliminary data were gathered to assess staff burnout and incidents of challenging behaviour before and after service changes.

The study was discussed with the Trust Lead for Research and Development. It was agreed that as service evaluation and development, all aspects of the process fell under the routine remit of the team psychologist. For this reason, formal ethical approval was not sought.

\section{Method}

\section{Setting and participants}

The Gatehouse is a 16-bed in-patient unit for adults with serious mental health problems and challenging behaviours. The majority of service users have a diagnosis of schizophrenia, and many have personality and/or substance misuse problems. The staff group consists of Mental Health Nurses, Health Care Support Workers and Occupational Therapists. Prior to the study, the Gatehouse was recording high levels of challenging behaviours and the management team were concerned both for patient care and that staff were at risk of burnout.

\section{Design}

This was an exploratory and naturalistic study in a clinical setting. The paper describes the formulation of team processes using the cognitive model, and interventions derived from this conceptualization. In addition, a simple before and after uncontrolled intervention design was used to gather preliminary data on the impact of service changes on incidents of challenging behaviour and staff burnout.

\section{Measures}

Routine Trust incident reports were collated to assess the range and frequency of challenging behaviours over the two time periods. The Maslach Burnout Inventory (MBI; Maslach, Jackson and Leiter, 1996) self-report measure was used to assess staff burnout at two time points. The MBI yields three subscales, each designed to assess a distinct aspect of "burnout syndrome": personal accomplishment (PA), emotional exhaustion (EE), and depersonalization (DP). The 22 items are rated on a 7-point scale ranging from 0 (never) to 6 (every day). The MBI has high internal consistency $(\alpha=.90$ for $\mathrm{EE}, \propto=.79$ for $\mathrm{DP}$, and $\propto=.71$ for PA) and adequate test-retest reliability over 6 months $(r=.59, .50$, and .63$)$. Eighteen out of 28 members of staff $(64.29 \%)$ returned completed questionnaires at the first time point, and 12 people returned questionnaires at the second (42.86\%). 


\section{Procedure and intervention}

Incidents of challenging behaviour were collated for an 8-month period. At the end of this time, all permanent clinical staff were sent the MBI by post and asked to complete the questionnaire to inform the management team about staff experience on the unit. Confidentiality was assured. Questionnaires were resent to any participant who had not returned the measure after 3 weeks.

Staff-service user relationships were formulated using the cognitive interpersonal model. This was informed by a range of interactions between the first author and those on the unit, including unit management meetings, staff supervision and many informal discussions with staff and service users on the unit. When complete, the conceptualization was used to inform discussions with the unit's management team, aimed at improving the experience of both residents and staff. Specific changes in service delivery (primarily through changes in staff behaviour) were identified on the basis of the formulation, and staff were encouraged and supported to engage in these over a 4-month period.

Incidents of challenging behaviour were then collated for a further 8-month period (including the time over which the team formulation was developed and service changes implemented). At the end of this time, the MBI was resent to all permanent clinical staff. Again, confidentiality was assured and questionnaires were resent to any participant who had not returned the measure after 3 weeks.

\section{Results}

\section{Team formulation and change process}

The cognitive interpersonal formulation was adapted to describe the staff-service user interactions, which were hypothesized to be contributing to the maintenance of service users' challenging behaviours and staff burnout. This indicated that the staff team often made negative, internal, global and stable attributions about residents and the function of their challenging behaviours. Many staff believed that they were not safe, and that others (e.g. senior clinicians and managers) did not care about them. With such beliefs, staff were understandably fearful and at times angry with others. These beliefs and feelings elicited avoidance of service users and at times led to inconsistent care planning and lack of adherence to best practice guidelines. As predicted by the model, residents responded reciprocally to staff behaviour, spending much time in their rooms, and relying on challenging behaviours to express distress and unmet needs. These behaviours in turn confirmed key staff beliefs on the unit, thereby maintaining the interpersonal cycle (see Figure 1).

The formulation was valuable to the extent that it led to workable interventions and change in staff behaviours. On the basis of the key beliefs and behaviours identified for staff and service users, and following a similar process to treatment planning in individual CBT, the staff team were encouraged to engage in the following:

(a) Improving residents' experiences by focusing on staff-service user interactions:

(i) encouraging staff to spend 1:1 time with residents each shift;

(ii) encouraging greater collaboration and active listening with service users;

(iii) encouraging daily activity planning in line with individuals' interests and goals; 


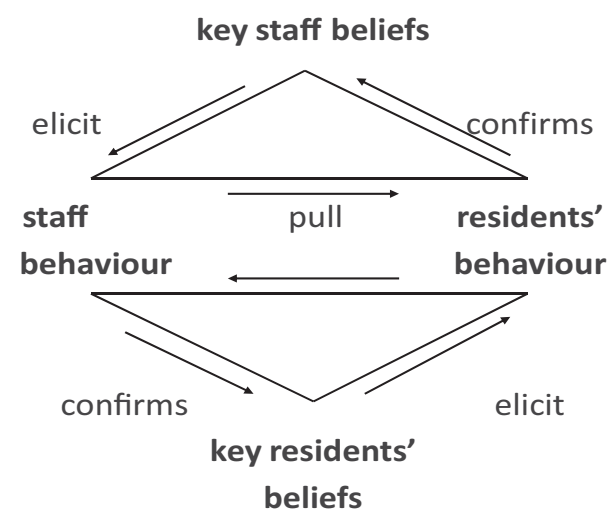

Key staff beliefs:

It's not safe here

Others (senior staff) don't understand what it's like here

Their (residents') behaviour is wilful

Key staff behaviours:

Avoid residents

Stay in the office
Key residents beliefs:

It's not safe here

Staff don't listen

Staff don't care

Key residents behaviours:

Spend time alone in own room

Increased expressions of distress

Increased challenging behaviours

Figure 1. The interpersonal cognitive model adapted for teams

(b) Building staff skills and confidence:

(i) providing training and ongoing supervision for staff on Recovery-based care;

(ii) providing brief but specific training on evidence based approaches (DBT principles, contingency management, and CBT conceptualization of voices and paranoia);

(c) Improving management and team working:

(i) developing and clarifying specific staff roles on the unit;

(ii) strengthening systems of accountability;

(iii) improving team morale and purpose through a team away day.

Direct observation and feedback from the management team indicated that many but not all staff engaged in changes in interaction, and attended in-house training sessions. Systems of accountability were highlighted and strengthened. The great majority of staff participated in the away day. We anticipated that these changes would improve relationships on the unit, and staff confidence and competence in working with challenging behaviours, thereby addressing the key staff and service user beliefs maintaining the problematic culture on the unit. For this reason, preliminary data on incidents of challenging behaviour and staff burnout were collected as secondary outcomes.

\section{Incidents of challenging behaviour}

Incidents of challenging behaviour were collected over two consecutive 8-month periods, the second including the period over which service changes were introduced. There was an overall 


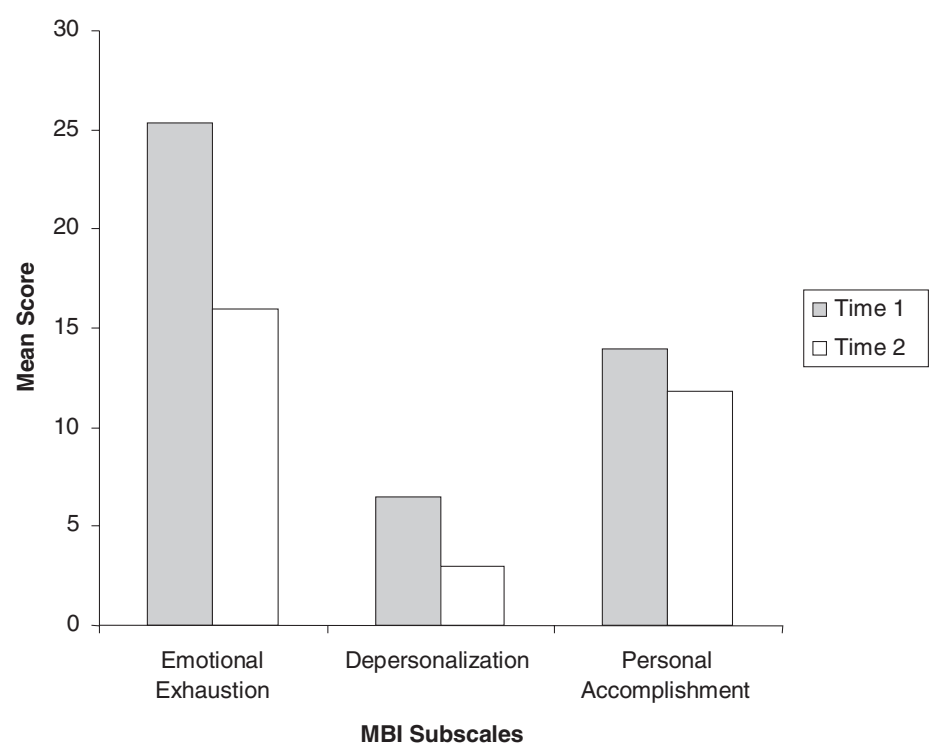

N.B. PA scale is reversed so low score $=$ high PA

Figure 2. MBI subscales before and after service changes

reduction in frequency of challenging behaviours of over one-third between the two time periods (124 to 78 incidents). This was mainly accounted for by reductions in the highest frequency behaviours: self-harm (40 to 14), verbal abuse to staff (23 to 18), absconding (20 to 14 ) and assaults on other patients/visitors (17 to 9). There were increases in physical assaults on staff (0 to 6), property damage (5 to 8 ), and racial abuse (0 to 1$)$. Overall, there was a significant reduction in challenging behaviours between the two time periods (two-tailed Fisher exact $p=.011)^{1}$

\section{Staff burnout}

Descriptive statistics for the MBI subscales are shown in Figure 2. It can be seen that, on average, levels of emotional exhaustion, depersonalization and personal accomplishment all improved over the course of the study. Unfortunately, it was not possible to compare these data statistically because members of the staff group changed over the period of the study. ${ }^{2}$

\section{Discussion}

This study explored the value of a cognitive approach to formulating team processes. Safran and Segal's (1996) cognitive interpersonal model was used to examine key staff-service user

\footnotetext{
${ }^{1}$ It should be noted that a number of service users moved on from the unit over the period of the study, and others were admitted.

${ }^{2}$ Nine members of staff left/joined the unit between the two phases of the study, leaving insufficient numbers for matched comparisons.
} 
interactions in a mental health in-patient setting, and inform management team planning. The formulation was effective in identifying service changes that were needed in order to improve care, and the staff team were then able to deliver these changes. Additionally, preliminary data indicate that staff burnout and incidents of challenging behaviours both reduced following these service changes.

Despite individual clinicians' best intentions, it is perhaps unsurprising that unexamined interpersonal processes often become problematic in mental health care settings. Team based consultation and formulation is now a key role for clinical psychologists and CBT clinicians, particularly those working in teams supporting people with serious and complex needs. If we are to work systemically to improve clinical outcomes through improved team working, we need to extend our formulation and intervention skills to these settings.

Prior to the intervention, staff at The Gatehouse reported levels of emotional exhaustion indicative of depleted emotion in relation to working with service users. Such a picture is perhaps familiar to people working in in-patient settings, with colleagues describing feeling overwhelmed and drained. It is understandable that these staff may become less willing and able to engage therapeutically with service users and instead withdraw to the nursing office, with interactions becoming less frequent and more functional. Furthermore, if we understand challenging behaviours as an expression of distress or unmet need, it makes sense that these behaviours would be linked to the nature and quality of interactions with staff. Supporting and improving the skills of the team could account for the improvements in both staff burnout and challenging behaviours found following the interventions, though it should be noted that causality cannot be inferred given our study design. Although there was a significant reduction in challenging behaviours overall between the two time periods, there were increases in some categories (physical assaults on staff, property damage and racial abuse). The reasons for this are unclear. It may be that this was related to staff and service user turnover, or that application of interventions focused more on higher frequency behaviours (self-harm, verbal abuse, absconding and assaults on other patients/visitors). It will be important to continue to monitor incidents to determine and make sense of enduring patterns of behaviour change.

As a naturalistic study, the design was not tightly controlled and there may be other reasons for improvements found in staff and service user measures. The time period described involved much change on the unit, and none of the changes would have been possible without the commitment and leadership of the unit manager. As a working unit, there was inevitably a degree of turnover in both staff and service users, and this may account for some of the changes observed. There were differences in staff response rates between the two time points, and we were unable to compare burnout indices statistically, and so cannot be certain that these improvements were significant. No attempt was made to link measures of staff burnout and challenging behaviours, and further work is needed to investigate the relationship between the two. While these issues certainly limit the weight and generalizability of our findings, we are also aware of the difficulties involved in developing controlled studies of team-based interventions. Within the limitations of the present study, however, these findings indicate that the cognitive interpersonal model can be usefully adapted to formulate problems within teams, and derive interventions likely to effect culture change. Our pilot data, although limited as described, indicate that cognitive approaches to team working may benefit both service users and staff. 


\section{Acknowledgements}

The authors would like to thank the staff at The Gatehouse, Nick Maguire and Tess Maguire for their statistical expertise, and Alice Brixey for her help with data management.

\section{References}

Carr, S. (2009) Leadership for health improvement - implementation and evaluation. Journal of Health Organisation and Management, 23, 200-211.

Cooperrider, D. and Whitney, D. (2005) Appreciative Inquiry: a positive revolution in change. San Francisco, CA: Berrett-Koehler Publishers.

Crawford, M., Adedeyi, T., Price, K. and Rutter, D. (2010) Job satisfaction and burnout among staff working in community based personality disorder services. International Journal of Social Psychiatry, 56, 196-206.

Currid, T. (2009). Experiences of stress among nurses in acute mental health settings. Nursing Standard, $23,40-46$.

Garman, A., Corrigan, P. and Morris, S. (2002). Staff burnout and patient satisfaction: evidence of relationships at the care unit level. Journal of Occupational Health Psychology, 7, 235-241.

Kowalski, K. (2008) Appreciative inquiry. Journal of Continuing Education in Nursing, 39, 104.

Lavender, T. and Hope, R. (2007). The End of the Beginning: new ways of working for applied psychologists in health and social care. Leicester: British Psychological Society.

Maslach, C., Jackson, S. E. and Leiter, M. P. (1996). Maslach Burnout Inventory Manual. Palo Alto, CA: Consulting Psychologists Press.

Maslach, C. and Leiter, M. P. (1997). The Truth about Burnout: how organisations cause personal stress and what to do about it. San Francisco, CA: Jossey-Bass.

Nelson, T., Johnson, S. and Bebbington, P. (2009). Satisfaction and burnout among staff of crisis resolution, assertive outreach and community mental health teams: a multicentre cross sectional survey. Social Psychiatry and Psychiatric Epidemiology, 44, 541-549.

O'Connell, M. and Stein, C. (2011). The relationship between case manager expectations and outcomes of persons diagnosed with schizophrenia. Community Mental Health Journal, 47, 424.

Onyett, S. (2007). Working Psychologically in Teams: new ways of working for applied psychologists in health and social care. Leicester: British Psychological Society.

Onyett, S. (2011) Revisiting job satisfaction and burnout in community mental health teams. Journal of Mental Health, 20, 198-209.

Safran, J. and Segal, Z. (1996). Interpersonal Processes in Cognitive Therapy (2 ${ }^{\text {nd }}$ edn). New York: Basic Books.

Tsai, J., Salyers, M. and McGuire, A. (2011). A cross sectional study of recovery training and staff attitudes in four community mental health centers. Psychiatric Rehabilitation, 34, 186. 